2. - Functions differentiable on the boundaries of regions, Ann. of Math. vol. 35 (1934) pp. 482-485.

3. — Differentiable manifolds, Ann. of Math. vol. 37 (1936) pp. 645-680.

4. - Differentiable functions defined in arbitrary subsets of Euclidean space, Trans. Amer. Math. Soc. vol. 40 (1936) pp. 309-317. Further references are given here.

5. H. O. Hirschfeld, Continuation of differentiable functions through the plane, Quart. J. Math. Oxford Ser. vol. 7 (1936) pp. 1-15.

6. M. R. Hestenes, Extension of the range of a differentiable function, Duke Math. J. vol. 8 (1941) pp. 183-192.

HARVARD UNIVERSITY

\title{
THE SYMMETRIC JOIN OF A COMPLEX
}

\section{E. CLARK}

1. The definition of $J$. Let $K$ be a polyhedron. With each pair of distinct points $p, q$ of $K$ we associate a closed line segment $p q$. No distinction is made between $p$ and $q$ and the corresponding end points of $p q$. The length of $p q$ is a continuous function of $p$ and $q$, and the length approaches zero if $p$ and $q$ approach a common limit. Distinct segments do not intersect except at a common end point. The points of these segments with their obvious natural topology make up $J$, the symmetric join of $K$. This space arises in [4] ${ }^{1}$ in connection with the problem of finding the chords of a manifold that are orthogonal to the manifold.

2. The subdivision of $J$. Let the mid-point of $p q$ be denoted by $\Lambda p \times q=\Lambda q \times p$, and let $p=\Lambda p \times p$. These points $\Lambda p \times q$ make up the symmetric product $S$ of $K$. Let the mid-point of the segment from $p$ to $\Lambda p \times q$ be denoted by $p \times q$, and let $p=p \times p$. These points $p \times q$ make up the topological product $P=K \times K$. Consider the closed segment of $p q$ from $p \times q$ to $q \times p$, it being understood that this segment is the point $p$ when $p=q$. All such segments form the "neighborhood" $N_{S}$. Clearly $N_{S}$ can be homotopically deformed in $N_{S}$ along the segments $p q$ upon $S$ with $S$ remaining pointwise invariant. Finally consider the closed segment of $p q$ from $p$ to $p \times q$, it being understood that this segment is the point $p$ when $p=q$. All such segments form the "neighborhood" $N_{K}$. Clearly $N_{K}$ can be homotopically deformed in $N_{K}$ along the segments $p q$ upon $K$ with $K$ remaining pointwise invariant.

Received by the editors July $23,1943$.

${ }^{1}$ Numbers in brackets refer to the References at the end of the paper. 
There is a natural cell division of $P$ whose oriented cells are $\sigma \times \tau$ for all oriented simplexes $\sigma, \tau$ of $K .^{2}$ Let $|\sigma \times \tau|$ denote the carrier of $\sigma \times \tau$, that is, the point set of $J$ associated with $\sigma \times \tau$. The rays $p q$ determine a mapping $\Lambda$ of $P$ on $S$. The cells $\Lambda|\sigma \times \tau|=\Lambda|\tau \times \sigma|$ form a natural cell division of $S .^{8}$ With this division $\Lambda$ is a cell mapping. Furthermore $\Lambda \sigma \times \tau$ is an orientation of $\Lambda|\sigma \times \tau|$ such that

$$
\Lambda \sigma^{s} \times \tau^{t}=(-1)^{s t} \Lambda \tau^{t} \times \sigma^{s},
$$

the superscripts denoting dimension. ${ }^{3}$

THEOREM 1. There is a simplicial division of $J$ such that $K, S, P$, $N_{S}$, and $N_{K}$ carry subcomplexes of the division, the first of which is a subdivision of the given polyhedron $K$, and the second and third of which are subdivisions of the above natural cell divisions of $S$ and $P$.

Proof. Let $[\sigma, \tau]$ be the point set made up of the points of all segments $p q$ with $p \in \sigma$ and $q \in \tau$. If $\sigma$ and $\tau$ have no common vertex, $[\sigma, \tau]=(\sigma, \tau)$, the join ${ }^{4}$ of $\sigma$ and $\tau$. In this case $S$ and $P$ separate $[\sigma, \tau]$ into four $(s+t+1)$-cells, where $s$ and $t$ are the dimensions of $\sigma$ and $\tau$, respectively.

Consider $[\sigma, \sigma]$. As an auxiliary set we construct the join $\left(\sigma, \sigma^{\prime}\right)$ with $\sigma^{\prime}$ homeomorphic to $\sigma$. Each pair of points, one in $\sigma$ and one in $\sigma^{\prime}$, determines their join in $\left(\sigma, \sigma^{\prime}\right)$. This join of the two points is called a ray of $\left(\sigma, \sigma^{\prime}\right)$. The mid-points of the rays form a set homeomorphic to $\sigma \times \sigma^{\prime}$ or $\sigma \times \sigma$. This product separates $\left(\sigma, \sigma^{\prime}\right)$ into two cells. We discard the one of these cells that contains $\sigma^{\prime}$ and retain $C$, the closure of the other cell which contains $\sigma, \sigma \times \sigma$, and rays connecting these two sets. We subdivide $\sigma \times \sigma$ into a simplicial complex in such a way that the set of points $p \times p$, all $p$ in $\sigma$, carries a subcomplex (that such a division exists is proved in [5]). Each simplex of this division is extended to a cell of $C$ by adding to the simplex the points of all rays of $C$ with one end point in the simplex (the set so obtained is a cell because it is obtained from a prism whose bases are simplexes by simple identifications in one base of the prism). These cells form a division of $C$ into a cell complex. The rays of $C$ that join $p$ and $p \times p$, all $p$ in $\sigma$, carry a subcomplex of this cell division. Let each such ray be reduced to a point by identification of all points of the ray. It is seen that the transform of $C$ under this identification is a cell complex which is a division of $[\sigma, \sigma] \cap N_{K}$.

2 The properties of $P$ which we use can be found in [3].

${ }^{3}$ See [5].

- A discussion of the join of two complexes is found in [3]. Also the properties of the join which we assume are presented in [1]. 
To obtain a division of $[\sigma, \sigma] \cap N_{S}$ we consider the prism $D$ defined as the product of $\sigma \times \sigma$ and a 1 -simplex. We use the same simplicial division of $\sigma \times \sigma$ used in the preceding paragraph. This division is extended to a cell division of $D$ by adding to each simplex the product of the simplex and the 1-simplex used to define $D$. One base of the prism $D$ is reduced to the symmetric product of $\sigma$ by the identification of $p \times q$ and $q \times p$ for all $p, q$ in $\sigma$. If our original simplicial division of $\sigma \times \sigma$ is properly determined (as in [5]), these identifications transform $D$ into a cell complex $E .^{3}$ Next the rays of $E$ which are images of the rays of $D$ with end points $p \times p$, all $p$ in $\sigma$, are reduced each to a point by identification. It is seen that the resulting space is a cell complex which is a division of $[\sigma, \sigma] \cap N_{S}$.

In the same way we subdivide $[\sigma, \tau]$ where $\sigma$ and $\tau$ have some but not all of their vertices in common. It is seen that Theorem 1 is true.

3. Homologies in $P$ and $S$. Let $z_{i}, i=1,2, \cdots$, be the cycles of a canonical basis ${ }^{5}$ for the chains of all dimensions of $K$, and let $f_{i}$ be the non-cyclic chains of this canonical basis. The range of the subscript of $f$ is a subset of the range of the subscript of $z$. For each $f$ there is a boundary relation $F f_{i}^{r+1}=e_{i} z_{i}^{\prime \prime}, e_{i} \geqq 1$. With each $z_{i}$ that is free there is associated an $e_{i}$ equal to zero.

A homology base for $P$ is given by the cycles $z_{i} \times z_{j}$ and $\left(1 /\left(e_{i}, e_{j}\right)\right) F\left(f_{i} \times f_{j}\right)$, the denominator of the coefficient denoting the greatest common divisor of $e_{i}$ and $e_{j}$. A complete set of homology relations for the cycles of this base is given by the two sets

$$
\left(e_{i}, e_{j}\right) z_{i} \times z_{j} \sim 0, \quad F\left(f_{i} \times f_{j}\right) \sim 0,
$$

with the understanding that $(a, 0)=a$.

We next obtain a homology base for $S$. We consider

$$
\Lambda z_{i}^{r} \times z_{j}^{s}=(-1)^{r s} \Lambda z_{j}^{s} \times z_{i}^{r}, \quad i \neq j,
$$

the equality holding because of (1). The dimension superscripts in (3) could be $r_{i}$ and $r_{j}$ rather than $r$ and $s$, but we use the less clear notation to simplify the printing. We also consider

$$
\begin{array}{cr}
\Lambda z_{i}^{r} \times z_{i}^{r}, & r \text { even, } \\
\Lambda \frac{1}{\left(e_{i}, e_{j}\right)} F\left(f_{i}^{r+1} \times f_{j}^{s+1}\right)=(-1)^{(r+1)(s+1)} \Lambda \frac{1}{\left(e_{i}, e_{j}\right)} F\left(f_{j}^{s+1} \times f_{i}^{r+1}\right), \\
i \neq j,
\end{array}
$$

5 See [3, p. 104]. 
and

$$
\frac{1}{2} \Lambda \frac{1}{e_{i}} F\left(f_{i}^{r+1} \times f_{i}^{r+1}\right)
$$

$r$ odd.

Using $[6$, p. 22, line 15] and the method of $[2, \S 4]$ we infer that the cycles (6) are integral, the orders of (3), (4), and (5) are $\left(e_{i}, e_{j}\right)$ while (6) have the orders $2 e_{i}$, the cycles (3), (4), (5), and (6) are a homology base for $S$, and the orders of these cycles are a complete set of homology relations in $S$.

4. The sum cycles and their group $S$. We shall apply the theory of the sum of two complexes to $J=N_{S}+N_{K}$. To prepare for this we define a sum cycle to be a cycle that is expressible as the sum of two cycles, one in $N_{S}$ and the other in $N_{K}$. The group of the sum cycles modulo those that bound in $J$ is called $S$. This group $S$ is known to be a subgroup of the Betti group of $J$. Here, as throughout the paper unless otherwise stated, we do not define a group for each dimension but combine all elements of all dimensions into a single group.

THEOREM 2. The group $S$ is generated by a free 0-dimensional element and a set of elements of order 2, each containing one and only one of the cycles (6).

Proof. Any cycle of $K$ can be deformed in $J$ onto a point $p$ of $K$ along the segments $p q$. Since $N_{K}$ is a deformation retract of $K$, the group of the cycles of $N_{K}$ modulo those that bound in $J$ has a single nonzero 0-dimensional element as generator.

A cycle of $S$ which bounds in $J$ is homologous in $N_{S}$ to a cycle of $P$. Indeed if $z=F C, z$ in $S, C$ in $J$, and $C$ simplicial, then $C$ can be expressed as the sum of two chains, one in $N_{S}$ and the other in $N_{K}$. The boundary of the first of these two chains is $z$ minus a cycle of $P$.

Any cycle $z$ of $P$ is homologous in $N_{S}$ to $\Lambda z$. Hence using (3), (4), (5), and (6) we obtain Theorem 2.

5. The seams and their group $\Gamma$. The seams are defined to be the cycles of $P$ that bound both in $N_{S}$ and $N_{K}$. We note that if $z$ is a cycle of $P$ and $z \sim 0$ in $N_{S}$, then since $z$ and $\Lambda z$ are homotopic, $\Lambda z \sim 0$ in $N_{S}$. But $N_{S}$ was shown in $\S 1$ to be a neighborhood retract of $S$. Hence $\Lambda z \sim 0$ in $S$. From this observation, from (1), and from $\$ 3$ we deduce that the cycles of $P$ that bound in $N_{S}$ are the cycles homologous in $P$ to all linear combinations of

$$
\left(z_{i}^{r} \times z_{j}^{s}\right)-(-1)^{r s}\left(z_{j}^{s} \times z_{i}^{r}\right), \quad i \neq j,
$$




$$
\begin{array}{cc}
z_{i}^{r} \times z_{i}^{r}, & r \text { odd, } \\
\frac{1}{\left(e_{i}, e_{j}\right)}\left[F\left(f_{i}^{r+1} \times f_{j}^{s+1}\right)-(-1)^{(r+1)(s+1)} F\left(f_{j}^{s+1} \times f_{i}^{r+1}\right)\right], & i \neq j
\end{array}
$$

and

$$
\frac{1}{e_{i}} F\left(f_{i}^{r+1} \times f_{i}^{r+1}\right)
$$

$r$ even.

To find the cycles of $P$ that bound in $N_{K}$ we consider the transformation $M(p \times q)=p$. We have $M|\sigma \times \tau|=|\sigma|$, the notation being that of $\$ 2$. Also for any set $A \subset P$ the discussion of $\$ 2$ implies that $A$ and $M(A)$ are homotopic in $N_{K}$. Hence $z_{i}^{r} \times z_{j}^{s}$ is homologous in $N_{K}$ to $M\left(z_{i}^{r} \times z_{j}^{s}\right)$ which is carried by $\left|z_{i}^{r}\right|$. Hence if $s>0, z_{i}^{r} \times z_{j}^{s}$ bounds in $N_{K}$ because it can be homotopically deformed into a set of dimension less than the dimension of the cycle.

Suppose $s=0$. We assume that $z_{i}^{0}$ is equal to 1 at exactly one vertex of $K$ and is equal to 0 at all other vertices. Then $M\left(z_{i}^{r} \times z_{j}^{0}\right)=z_{l}^{r}$ because $z_{i}^{r} \times z_{j}^{0}$ is obtained by sliding $z_{i}^{r}$ along the rays of a cone.

If in $f_{j}^{s+1}$ the exponent $s$ is greater than $0,\left(1 /\left(e_{i}, e_{j}\right)\right) F\left(f_{i}^{r+1} \times f_{j}^{s+1}\right)$ $\sim 0$ in $N_{K}$ because it is homotopic to a cycle carried by $\left|f_{i}^{r+1}\right|$, and the dimension of the cycle is $r+s+1$ which is greater than $r+1$. If $s=0$, the same homology holds because $e_{j}=1$.

We have shown that the cycles of $P$ that bound in $N_{K}$ are the cycles homologous in $P$ to all linear combinations of

and

$$
\begin{array}{cc}
z_{i}^{r} \times z_{j}^{*}, & s>0, \\
z_{i}^{r} \times z_{1}^{0}-z_{i}^{r} \times z_{j}^{0}, & i \neq 1,
\end{array}
$$

$$
\frac{1}{\left(e_{i}, e_{j}\right)} F\left(f_{i}^{r+1} \times f_{i}^{s+1}\right) \text {. }
$$

It is easily seen that if one cycle of a homology class of the Betti group of $P$ is a seam, then all cycles of the class are seams. Furthermore such classes form a subgroup of the Betti group. This subgroup of the Betti group of $P$ made up of the homology classes whose cycles are seams is called $\Gamma$. Combining the results of this section we obtain the following theorem.

THEOREM 3. The subgroup $\Gamma$ is generated by the homology classes that contain (8), (9), (10),

$$
\text { (7) with } r>0 \text { and } s>0 \text {, }
$$

and

$$
\left(z_{i}^{r} \times z_{1}^{0}\right)-\left(z_{i}^{r} \times z_{j}^{0}\right)-\left(z_{1}^{0} \times z_{i}^{r}\right)+\left(z_{j}^{0} \times z_{i}^{r}\right), \quad i \neq 1 .
$$


6. The Betti group $B(J)$ of $J$. The theory of the sum of two complexes $^{6}$ gives the isomorphism $B(J) / S \cong \Gamma$. However we shall obtain a sharper result.

For each seam $z$ we shall define a pair of singular chains $c$ of $N_{S}$ and $d$ of $N_{K}$ such that $F c=F d=z$. To define $c$ we deform $z$ along the segments $p q$ from $P$ to $S$ sweeping out the continuous image of a prism whose bases are $|z|$ and $|\Lambda z|$. This deformation gives rise to a singular chain $c$ whose boundary ${ }^{7}$ is $z-\Lambda z$. But since $z$ is a seam it follows from (1), (8), (9), (10), (11), and (12) that $\Lambda z=0$. In a similar way we define the chain $d$ of $N_{K}$.

Let $H(z)$ be the homology class of $B(J)$ which contains $c-d$. We know that any element of the Betti group $B(J)$ is expressible as the sum of an element of $S$ and an $H(z), z$ a seam. ${ }^{6}$ Hence to characterize $B(J)$ we need only find the relations that involve the $H(z)$. We know that any homology among the $H(z)$ implies the same homology in $P$ among the $z$. On the other hand, $z \sim 0$ in $P$ implies $^{6}$ that $H(z)$ is in $S$. We shall examine all relations $z \sim 0$ in $P$, and find the corresponding relations among the $H(z)$. The relations to examine are (2) applied to (8), (9), (10), (11), and (12).

First we consider $\left(e_{i}, e_{j}\right)(11) \sim 0$ in $P$. We consider

$$
\begin{aligned}
C= & \alpha\left[\left(f_{i}^{r+1} \times z_{j}^{s}\right)-(-1)^{s(r+1)}\left(z_{j}^{s} \times f_{i}^{r+1}\right)\right] \\
& +\beta\left[(-1)^{r}\left(z_{i}^{r} \times f_{j}^{s+1}\right)-(-1)^{r s}\left(f_{j}^{s+1} \times z_{i}^{r}\right)\right],
\end{aligned}
$$

with $\alpha, \beta$ integers such that $\alpha e_{i}+\beta e_{j}=\left(e_{i}, e_{j}\right)$. Since we find in [3, p. $138,(5.5)]$ that

$$
F\left(x^{p} \times y\right)=\left(F x^{p} \times y\right)+(-1)^{p}\left(x^{p} \times F y\right),
$$

it follows that $F C=\left(e_{i}, e_{j}\right)(11)$. Next we deform $C$ along the segments $p q$ from $P$ to $S$ sweeping out the continuous image of a prism whose bases are $C$ and $\Lambda C$. This deformation gives rise to a singular chain ${ }^{7}$ whose boundary is $\Lambda C-C-\left(e_{i}, e_{j}\right) c$ because, as defined above, $\left(e_{i}, e_{j}\right) c$ is swept out by $F C=\left(e_{i}, e_{j}\right) z$. Using (1) we calculate that $\Lambda C=0$. Hence $\left(e_{i}, e_{j}\right) c+C$ bounds in $N_{S}$. In the same way it is seen that $\left(e_{i}, e_{j}\right) d+C$ bounds in $N_{K}$. Hence $\left(e_{i}, e_{j}\right)(c-d) \sim 0$ in $J$ and $\left(e_{i}, e_{j}\right) H(z)=0$.

In the same way we show that $e_{i} H(12)=\left(e_{i}, e_{j}\right) H(9)=e_{i} H(10)=0$. The argument is as above with (13) replaced by

- See $[3$, p. 267, 18].

${ }^{7}$ See $[3$, p. $307,(21.2)]$. 


$$
\begin{aligned}
& C=\left(f_{i}^{r+1} \times z_{1}^{0}\right)-\left(f_{i}^{r+1} \times z_{j}^{0}\right)-\left(z_{1}^{0} \times f_{i}^{r+1}\right)+\left(z_{j}^{0} \times f_{i}^{r+1}\right), \\
& C=\left(f_{i}^{r+1} \times f_{j}^{s+1}\right)-(-1)^{(r+1)(s+1)}\left(f_{j}^{s+1} \times f_{i}^{r+1}\right)
\end{aligned}
$$

and

$$
C=\left(f_{i}^{r+1} \times f_{i}^{r+1}\right)
$$

respectively.

Finally we consider $e_{i}\left(z_{i}^{r} \times z_{i}^{r}\right) \sim 0$ in $P, r$ odd. Take $C=\left(f_{i}^{r+1} \times z_{i}^{r}\right)$. Then $F C=e_{i}\left(z_{i}^{r} \times z_{i}^{r}\right)$. Using (14), (1), and the fact that $r$ is odd, we compute that

$$
\Lambda F\left(f_{i}^{r+1} \times f_{i}^{r+1}\right)=2 \Lambda\left(f_{i}^{r+1} \times z_{i}^{r}\right) .
$$

Hence

$$
e_{i} H\left(z_{i}^{r} \times z_{i}^{r}\right) \supset(1 / 2) \Lambda F\left(f_{i}^{r+1} \times f_{i}^{r+1}\right) .
$$

This implies that the elements of $S$ with dimension greater than zero are in the subgroup of $B(J)$ generated by the $H(z), z$ a seam. Also $H\left(z_{i}^{r} \times z_{i}^{2}\right), r$ odd, is of order $2 e_{i}$. Our discussion implies the following:

TheOREM 4. The Betti group $B(J)$ is generated by a nonzero 0-dimensional element, $H(8), H(9), H(10), H(11)$, and $H(12)$; furthermore $a$ complete set of relations for these generators consists of $2 e_{i} H(8)$ $=\left(e_{i}, e_{j}\right) H(9)=e_{i} H(10)=\left(e_{i}, e_{j}\right) H(11)=e_{i} H(12)=0$.

We shall conclude with two corollaries of Theorem 4 . Let $K_{a}$ be $K$ augmented as defined in $\left[3\right.$, p. 130]. Let $B\left(K_{a}\right)$ be generated by $U_{i}$, $i=1,2, \cdots$, and $u_{k}, k=1,2, \cdots$, where the $U_{i}$ are free and independent, $u_{k}$ is of order $e_{k}$, and the $e_{k}$ are the torsion coefficients. Let the dimensions of $U_{i}$ and $u_{k}$ be $r_{i}$ and $r_{k}$, respectively. Let $B^{n}$ denote the $n$-dimensional Betti group of $J$.

CoRollary 1. To each pair $U_{i}$ and $U_{j}, i \neq j, r_{i}+r_{j}=n-1$, there corresponds a free element of $B^{n}$; to each $U_{i}, r_{i}=(n-1) / 2, r_{i}$ odd, there corresponds a free element of $B^{n}$; to each pair $u_{k}$ and $u_{l}, k \neq l, r_{k}+r_{l}$ $=$ either $n-1$ or $n-2$, there corresponds an element of $B^{n}$ of order $\left(e_{k}, e_{l}\right)$; to each $u_{k}, r_{k}=(n-1) / 2, r_{k}$ odd, there corresponds an element of $B^{n}$ of order $2 e_{k}$; and to each $u_{k}, r_{k}=(n-2) / 2, r_{k}$ even, there corresponds an element of $B^{n}$ of order $e_{k}$; the elements mentioned generate $B^{n}$, and their orders are a complete set of relations for these generators; finally $B^{0}$ is a free cyclic group of rank 1.

Let $R^{n}$ and $R^{n}(J)$ denote the $n$-dimensional Betti numbers of $K_{a}$ and $J$, respectively. 
COROLlary 2. We have

$$
R^{n}(J)=\sum_{i} R^{i} R^{n-i-1}+A^{n},
$$

with the summation over all integers $i$ from 0 to the greatest integer less than $(n-1) / 2$ and

$$
A^{n}= \begin{cases}R^{(n-1) / 2}, & (n-1) / 2 \text { an odd integer, } \\ 0, & (n-1) / 2 \text { not an odd integer. }\end{cases}
$$

\section{REFERENCES}

1. C. E. Clark, On the join of two complexes, Bull. Amer. Math. Soc. vol. 49 (1943) pp. 126-129.

2. - The Betti groups of symmetric and cyclic products, Bull. Amer. Math. Soc. vol. 49 (1943) pp. 450-454.

3. S. Lefschetz, Algebraic topology, Amer. Math. Soc. Colloquium Publications vol. 27 (1942).

4. M. Morse, The calculus of variations in the large, Amer. Math. Soc. Colloquium Publications vol. 18 (1934).

5. M. Richardson, On the homology characters of symmetric products, Duke Math. J. vol. 1 (1935) pp. 50-69.

6. -, Special homology groups, Proc. Nat. Acad. Sci. U. S. A. vol. 24 (1938) pp. 21-23.

Bradley Polytechnic Institute 